\title{
Blind separation of complex-valued satellite-AIS data for marine surveillance: a spatial quadratic time-frequency domain approach
}

\author{
Omar Cherrak $^{1}$, Hicham Ghennioui ${ }^{2}$, Nadège Thirion Moreau ${ }^{3}$, El Hossein Abarkan ${ }^{4}$ \\ ${ }^{1}$ LREA, Institut supérieur du Génie Appliqué, Maroc \\ ${ }^{1,2,4}$ LSSC, Université Sidi Mohamed Ben Abdellah, Faculté des Sciences et Techniques, Maroc \\ ${ }^{3}$ Aix-Marseille Université, CNRS, France \\ ${ }^{3}$ Université de Toulon, France
}

\begin{tabular}{l}
\hline \hline Article Info \\
\hline Article history: \\
Received Jun 30, 2018 \\
Revised Nov 21, 2018 \\
Accepted Des 15, 2018
\end{tabular}

Keywords:

Blind source separation

Joint zero-(block)

diagonalization

Marine surveillance

Matrix decompositions

Satellite-automatic

identification system

Spatial generalized mean

ambiguity function

Spatial time-frequency based

approach

\begin{abstract}
In this paper, the problem of the blind separation of complex-valued Satellite-AIS data for marine surveillance is addressed. Due to the specific properties of the sources under consideration: they are cyclo-stationary signals with two close cyclic frequencies, we opt for spatial quadratic time-frequency domain methods. The use of an additional diversity, the time delay, is aimed at making it possible to undo the mixing of signals at the multi-sensor receiver. The suggested method involves three main stages. First, the spatial generalized mean Ambiguity function of the observations across the array is constructed. Second, in the Ambiguity plane, Delay-Doppler regions of high magnitude are determined and Delay-Doppler points of peaky values are selected. Third, the mixing matrix is estimated from these Delay-Doppler regions using our proposed non-unitary joint zero-(block) diagonalization algorithms as to perform separation.
\end{abstract}

Copyright (c) 2019 Institute of Advanced Engineering and Science. All rights reserved.

\footnotetext{
Corresponding Author:

Omar Cherrak,

LREA, Institut supérieur du Génie Appliqué,

279 Bd Bir Anzarane, Casablanca, Maroc.

omar.cherrak@iga.ac.ma
}

\section{INTRODUCTION}

This paper concerns the spatial automatic identification system (S-AIS) dedicated to marine surveillance by satellite. It covers a larger area than the terrestrial automatic identification system [1], [2]. The idea of switching to satellite monitoring was introduced because of the fast and dynamic development of the marine traffic [3-5]. It was an emergency to adopt a method that operates a global monitoring with reliability, efficiency and robustness. However, this generalization to space involves several phenomena. Among these phenomena, we found:

(a) The speed of the satellite movement generates the Doppler effect which creates frequency offsets at the S-AIS signals [6],

(b) The propagation delay of the signals and their attenuation due to the satellite altitude [7],

(c) When a wide area is covered by the satellite, it certainly includes several traditional AIS cells. In fact, the time propagation of signals transmitted from vessels to the satellite vary according to the position 
of the ships and the maximum coverage area of the satellite antenna. Due to these two problems, it mainly affects the organizational mechanism of S-AIS signals. It results a collision data, as illustrated in the Figure 1, issued by vessels located in different AIS cells but received at the antenna of the same satellite [8], [9]. For this reason, we present new approaches to address this problem where the Doppler effect and the propagation delay are also taken into consideration.

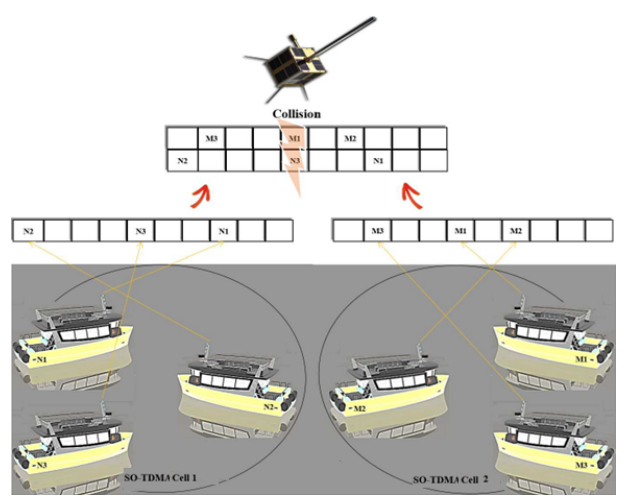

Figure 1. Collision problem: The AIS signals from two different SO-TDMA cells received to the satellite antenna at the same time.

In fact, to solve the collision problem, few works have focused on blind separation of sources (BSS) methods [10], [11]. In [11], Zhou et al. present a multi-user receiver equipped with an array of antennas embedded in Low Orbit Earth (LEO) satellite. The principle of this receiver is to exploit spatial multiplexing in a non-stationary asynchronous context. Indeed, the authors consider the equation below:

$$
\mathbf{X}=\mathbf{H G}(\mathbf{S} \odot \Phi)+\mathbf{N}
$$

where $\odot$ is the Schur-Hadamard operator, $\mathbf{X}=\left[\mathbf{x}_{1}, \mathbf{x}_{2}, \ldots, \mathbf{x}_{P N}\right] \in \mathbb{C}^{M \times P N}, \mathbf{x}_{n}=\mathbf{x}\left(n T_{s}\right), 1 \leq n \leq P N$, is the observation matrix, $\mathbf{H}=\left[\mathbf{h}_{1}, \ldots, \mathbf{h}_{d}\right] \in \mathbb{C}^{M \times d}$ is the matrix of antenna response, $\mathbf{G}=\operatorname{diag}\left\{g_{1}, g_{2}, \ldots, g_{d}\right\}$ $\in \mathbb{R}^{d \times d}$ contains the power of the sources, $\mathbf{S}=\left[\mathbf{s}_{1}^{H}, \mathbf{s}_{2}^{H}, \ldots, \mathbf{s}_{d}^{H}\right]^{H} \in \mathbb{C}^{d \times P N}$ is the matrix of sources and

$$
\Phi=\left(\begin{array}{cccc}
1 & \varphi_{1}^{1} & \ldots & \varphi_{1}^{P N-1} \\
1 & \varphi_{2}^{1} & \ldots & \varphi_{2}^{P N-1} \\
\vdots & \vdots & \ddots & \vdots \\
1 & \varphi_{d}^{1} & \ldots & \varphi_{d}^{P N-1}
\end{array}\right),
$$

where $\varphi_{k}=e^{j 2 \pi \Delta f_{k} T_{s}}$ contains the Doppler frequencies of the sources. The principle of this method is based on joint diagonalization (JD) of matrices in order to reconstruct the S-AIS sources from separation matrix estimation [12]. However, because of the very specific properties of the S-AIS signals (complex and cyclo-stationary with two close cyclic frequencies), we opt for spatial quadratic time-frequency domain methods. Our aim is reshaping the collision problem into BSS problem more simpler than (1). We will show how another type of decomposition matrix named joint zero-diagonalization (JZD) of matrices set resulting from spatial quadratic time-frequency distributions allows the restitution of S-AIS sources.

\section{TRANSMISSION SCHEME}

\subsection{AIS Frame}

The AIS frame is a length of 256 bits and occupies one minute. It is divided into 2250 time slots where one slot equals $26.67 \mathrm{~ms}$ [13]. Its structure as illustrated in Figure 2 contains a training sequence (TS) consisting zero and one which takes 24 bits. The start flag (SF) and the end flag (EF) for information takes 8 bits. A Frame Check Sequence (FCS) (or 16 bits Cyclic Redundancy Code (CRC)) is added to the data information (168 bits) in which a zero is inserted after every five continuous one. The binary sequence $\left\{a_{k}\right\}_{0 \leq k \leq K}$ of the AIS frame takes the values $\{-1,+1\}$ since the NRZI encoding is used. Moreover, the modulation specified 
by S-AIS standard is Gaussian Minimum Shift Keying (GMSK) [14]. The encoded message is modulated and transmitted at $9600 \mathrm{bps}$ on $161.975 \mathrm{MHz}$ and $162.025 \mathrm{MHz}$ frequencies carrier.

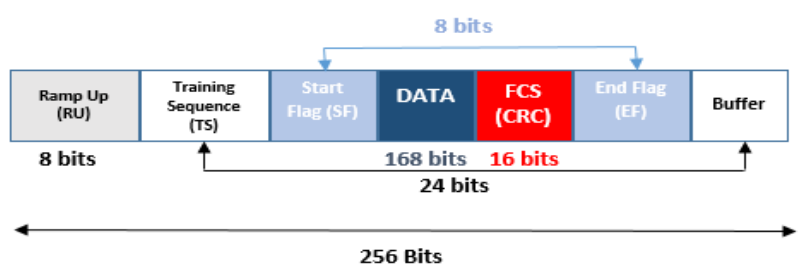

Figure 2. AIS Frame.

\subsection{GMSK modulation}

The resulting sequence after the bit stuffing and NRZI coding procedure is modulated with GMSK which is a frequency-shift keying modulation producing constant-envelope and continuous-phase. Hence, the signal can be written as $s_{g}(t)=\sum_{k=0}^{+\infty} a_{k} g\left(t-k T_{s}\right)$, where $a_{k}$ are the transmitted symboles, $T_{s}$ is the symbol period and $g(t)=\sqrt{\frac{2 \pi}{\log 2}} B \exp \left(-\frac{2}{\log 2}(\pi B t)^{2}\right)$ represents the shaping Gaussian filter where $B$ is the bandwidth of the Gaussian filter. The GMSK modulation is described by the bandwidth-time (BT) product where S-AIS uses BT $=0.4$ and $T_{s}=\frac{1}{9600} s$ ). Making the signal on one of the frequencies carrier $f_{c}$, produces a signal of spectral characteristic which is adapted to the band-pass channel transmission. The GMSK signal is, thus, expressed as : $s(t)=\Re\left\{e^{-j\left(2 \pi f_{c} t+\phi(t)\right)}\right\}=I(t) \cos \left(2 \pi f_{c} t\right)-Q(t) \sin \left(2 \pi f_{c} t\right)$, where $\Re\{\cdot\}$ is the real part of a complex number, $\phi(t)=2 \pi h \sum_{k=0}^{+\infty} a_{k} g\left(t-k T_{s}\right)$ is the instantaneous phase of $s_{g}(t)$ where, in the AIS system, the modulation index is theoretically equal to $h=0.5$ [15], $I(t)$ (resp. $Q(t)$ ) modulates the frequency carrier in phase (resp. in phase quadrature). All steps of the GMSK modulation can be presented in the Figure 3.

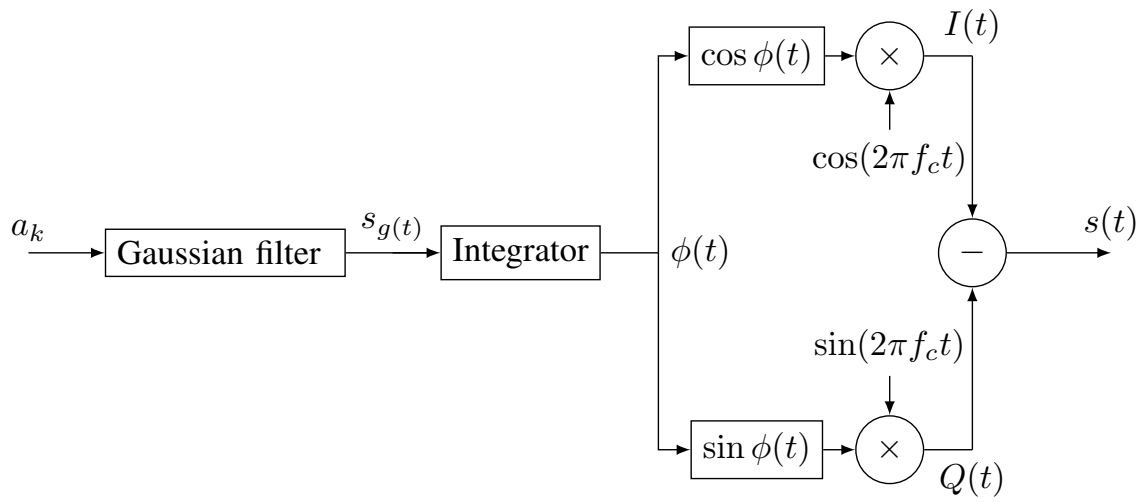

Figure 3. GMSK modulator scheme.

\section{PROBLEM STATEMENT: COLLISION \& BSS IN INSTANTANEOUS CONTEXT}

\subsection{Mathematical model of collision problem}

The collision problem can be simply expressed as follows:

$$
\mathbf{x}(t)=\sum_{j=1}^{J} h_{j} s_{j}\left(t-\tau_{j}\right) e^{-i 2 \pi \Delta f_{j} t}+\mathbf{n}(t),
$$

where $\mathbf{x}(t)$ is the received signal by the satellite, $s_{j}$ is the transmitted signal by the $j-t h$ vessel, $h_{j}, \tau_{j}$ and $\Delta f_{j}$ are respectively the coefficients of the channel, the delay and the Doppler shift corresponding to the $j-t h$ vessel with $J$ is the number of vessels and $\mathbf{n}(t)$ is an additive stationary white Gaussian noise, mutually uncorrelated, independent from the $s_{j}$, with the variance $\sigma_{\mathbf{n}}^{2}$.

\subsection{Reshape the collision problem into BSS problem}

We show, here, that (2) can be written in BSS nomenclature in which the delay and the Doppler shift caused by the satellite speed are considered. However, before any reformulation, we notice that the mixing 
matrix considered for S-AIS application is an instantaneous mixture due to the absence of obstacles in the ocean. Thus, we set $J=n$, the collision problem can be easily modeled in a BSS problem as follows:

$$
\mathbf{x}(t)=\mathbf{H} \mathbf{s}(t)+\mathbf{n}(t),
$$

where $\mathbf{H}$ is a $(m \times n)$ mixing matrix, $\mathbf{s}(t)=\left[s_{1}(t), s_{2}(t), \ldots, s_{n}(t)\right]^{T}$ is a $(n \times 1)$ sources vector with $s_{j}(t)=$ $s_{j}\left(t-\tau_{j}\right) \exp \left\{-i 2 \pi \Delta f_{j} t\right\}, \forall j=1, \ldots, n$ and $\mathbf{x}(t)=\left[x_{1}(t), x_{2}(t), \ldots, x_{m}(t)\right]^{T}, \mathbf{n}(t)$ $=\left[n_{1}(t), n_{2}(t), \ldots, n_{m}(t)\right]^{T}$ are respectively the $(m \times 1)$ observations and noises vectors. The superscript $(.)^{T}$ denotes the transpose operator. Our developments are based on the following assumptions:

Assumption A: The noises $n_{j}(t)$ for all $j=1, \ldots, m$ are stationary, white, zero-mean, mutually uncorrelated random signals and independent from the sources with variance $\sigma_{\mathbf{n}}^{2}$.

Assumption B: For each $s_{j}$ of the $n$ sources, there Delay-Doppler points of only one source is present in the Ambiguity plane.

Assumption C: The number of sensors $m$ and the number of sources $n$ are both known and $m \geq n$ to deal with an over-determined model (the under-determined case is outside of the scope in this paper).

\section{PRINCIPLE OF THE PROPOSED METHODS BASED ON THE SPATIAL GENERALIZED MEAN AMBIGUITY FUNCTION}

We show, here, how the algorithms proposed in [16], [17] adresses the problem of the separation of instantaneous mixtures of S-AIS data. The principle of the proposed methods are based on three main steps: first, the SGMAF of the observations across the array is constructed. Second, in the Ambiguity plane, DelayDoppler regions of high magnitude are determined and Delay-Doppler points of peaky values are selected. Third, the mixing matrix is estimated from these Delay-Doppler regions so as to perform separation and to undo the mixing of signals at the multi-sensor receiver.

\subsection{The Spatial Generalized Mean Ambiguity Function}

With regard to BSS, it has been shown that spatial time-frequency distributions are an effective tool when signature of the sources differ in certain points of the time-frequency plan [18]. However, in the cyclostationary sources case, the Delay-Doppler frequency domain seems to be a more natural field for the reestimation of sources than the time-frequency domain. As mentioned in [19], the approaches based on information derived from spatial Ambiguity function (SAF) or on SGMAF should be used. In fact, for any vectorial complex signal $\mathbf{z}(t)$, the SGMAF is expressed as [20-22]:

$$
\overline{\mathbf{A}}_{\mathbf{z}}(\nu, \tau)=\int_{-\infty}^{\infty} \mathbf{r}_{\mathbf{z}}(t, \tau) e^{-j 2 \pi \nu t} d t=\mathrm{E}\left\{\left\langle\mathbf{z}, \mathbf{s}_{\tau, \nu} \mathbf{z}\right\rangle\right\}
$$

where $\left(\mathbf{s}_{\tau, \nu} \mathbf{z}\right)$ is the operator of elementary Delay-Doppler translations of $\mathbf{z}$ defined by $\left(\mathbf{s}_{\tau, \nu} \mathbf{z}\right)(t)$ $=\mathbf{z}(t-\tau) e^{j 2 \pi(t-\tau) \nu}$ and $\mathbf{r}_{\mathbf{z}}(t, \tau)=\mathbf{R}_{\mathbf{z}}\left(\left(t+\frac{\tau}{2}\right),\left(t-\frac{\tau}{2}\right)\right)=\mathrm{E}\left\{\mathbf{z}\left(t+\frac{\tau}{2}\right) \mathbf{z}^{H}\left(t-\frac{\tau}{2}\right)\right\}$, where $\mathbf{R}_{\mathbf{z}}(t, \tau)$ stands for the correlation matrix of $\mathbf{z}(t), \mathbf{E}\{$.$\} stands for the mathematical expectation operator and superscript$ $(.)^{H}$ denotes the conjugate transpose operator. $\overline{\mathbf{A}}_{\mathbf{z}}(\nu, \tau)$ characterizes the average correlation of all pairs separated by $\tau$ in time and by $\nu$ in frequency [21], [22]. Notice that the diagonal terms of the matrix $\overline{\mathbf{A}}_{\mathbf{z}}(\nu, \tau)$ are called auto-terms, while the other ones are called cross-terms.

\subsection{Selection of peaky Delay-Doppler points}

Under the linear data model in (3), the SGMAF of observations across the array at a given DelayDoppler point is a $(m \times m)$ matrix admits the following decomposition:

$$
\begin{aligned}
\overline{\mathbf{A}}_{\mathbf{x}}(\nu, \tau) & =\mathbf{H} \overline{\mathbf{A}}_{\mathbf{s}}(\nu, \tau) \mathbf{H}^{H}+\overline{\mathbf{A}}_{\mathbf{n}}(\nu, \tau), \\
& =\mathbf{H} \overline{\mathbf{A}}_{\mathbf{s}}(\nu, \tau) \mathbf{H}^{H}+\mathbf{R}_{\mathbf{n}}(\tau),
\end{aligned}
$$

where $\overline{\mathbf{A}}_{\mathbf{S}}(\nu, \tau)$ represents the $(n \times n)$ SGMAF of sources defined similarly to $\overline{\mathbf{A}}_{\mathbf{z}}(\nu, \tau)$ in (4) and $\mathbf{R}_{\mathbf{n}}(\tau)=$ $\sigma_{\mathbf{n}}^{2} \alpha(\tau) \mathbf{I}_{m}$ with $\alpha(\nu)=\int_{-\infty}^{\infty} e^{-j 2 \pi \nu t} d t$ and $\mathbf{I}_{m}$ is the $m \times m$ identity matrix. It is known that the matrix $\overline{\mathbf{A}}_{\mathbf{s}}(\nu, \tau)$ for any $\tau$ and $\nu$ has no special structure. However, there are some Delay-Doppler points where this matrix has a specific algebraic structure :

(a) Diagonal, for points where each of them corresponds to a single auto-source term for all source signals, 
(b) Zero-diagonal for points where each of them correspond to all two by two cross-source term (this structure is exploited because the signature of the sources differ in certain points of the Delay-Doppler plan on the zero-diagonal part (as shown in section 5.).

Our aim is to take advantage of these properties of the $\overline{\mathbf{A}}_{\mathbf{x}}(t, \nu)$ in (5) since the element of this is no more (zero) diagonal matrices due to the mixing effect in order to estimate the separation matrix $\mathbf{B}$ (the pseudo-inverse of matrix $\mathbf{H}$ ) and restore the unknown sources.

\subsection{Construction of $\mathcal{M}$ (set of Delay-Doppler matrices of the observations across the array at the cho- sen Delay-Doppler points)}

We use the detector suggested in [23] (denoted $C_{\text {Ins }}$ ) for the instantaneous mixture considered without pre-whitening of the observations. The idea is to find "useful" Delay-Doppler points which consists in keeping Delay-Doppler points with a sufficient energy, then using the rank-one property to detect single cross-source terms (we don't make any assumptions on the knowledge of cyclic frequencies) in the following way:

$$
\left\{\begin{array}{l}
\left\|\overline{\mathbf{A}}_{\mathbf{x}}(t, \nu)\right\|>\epsilon_{1}, \\
\frac{\lambda_{\max }\left\{\overline{\mathbf{A}}_{\mathbf{x}}(t, \nu)\right\}}{\left\|\overline{\mathbf{A}}_{\mathbf{x}}(t, \nu)\right\|}-1>\epsilon_{2},
\end{array}\right.
$$

where $\epsilon_{1}, \epsilon_{2}$ are (sufficiently) small positif values and $\lambda_{\max }\{$.$\} is the largest eigenvalue of a matrix.$

\subsection{Non-unitary joint zero-(block) diagonalization algorithms (NU - JZ(B)D)}

The matrices belonging to the set $\mathcal{M}$ (whose size is denoted by $N_{m}\left(N_{m} \in \mathbb{N}^{*}\right)$ ) all admit a particular structure since they can be decomposed into $\mathbf{H} \overline{\mathbf{A}}_{\mathbf{s}}(\nu, \tau) \mathbf{H}^{H}$ with $\overline{\mathbf{A}}_{\mathbf{s}}(\nu, \tau)$ a zero-diagonal matrix with only one non null term on its zero-diagonal. One possible way to recover the mixing matrix $\mathbf{B}$ is to directly joint zerodiagonalize the matrix set $\mathcal{M}$. It has to be noticed that the recovered sources (after multiplying the observations vector by the estimated matrix B) are obtained up to a permutation (among the classical indetermination of the BSS). Hence, two BSS methods can be derived. The first called JZD CG $_{D D}$ algorithm based on conjugate gradient approach [16]. The second JZD $\mathrm{LM}_{\mathrm{DD}}$ algorithm based on Levenbreg-Marquardt scheme [17].

To tackle that problem, we propose here, to consider the following cost function [16], [17], $\mathcal{C}_{Z B D}(\mathbf{B})=$ $\sum_{i=1}^{N_{m}}\left\|\operatorname{Bdiag}_{(\mathbf{n})}\left\{\mathbf{B M}_{i} \mathbf{B}^{H}\right\}\right\|_{F}^{2}$, where the matrix operator $\operatorname{Bdiag}_{(\mathbf{n})}\{$.$\} is defined as follows:$

$$
\operatorname{Bdiag}_{(\mathbf{n})}\{\mathbf{M}\}=\left(\begin{array}{cccc}
\mathbf{M}_{11} & \mathbf{0}_{12} & \ldots & \mathbf{0}_{1 r} \\
\mathbf{0}_{21} & \mathbf{M}_{22} & \ddots & \mathbf{0}_{2 r} \\
\vdots & \ddots & \ddots & \vdots \\
\mathbf{0}_{r 1} & \mathbf{0}_{r 2} & \ldots & \mathbf{M}_{r r}
\end{array}\right)
$$

where $\mathbf{M}$ is a $N \times N\left(N=n\left(L+L^{\prime}\right)\right.$ where $L$ is the order of the FIR filter and $L^{\prime}$ is the number of delays considered when the convolutif mixture is considered) square matrix whose block components $\mathbf{M}_{i j}$ for all $i, j=1, \ldots, r$ are $n_{i} \times n_{j}$ matrices (and $n_{1}+\ldots+n_{r}=N$ ) denoting by $\mathbf{n}=\left(n_{1}, n_{2}, \ldots, n_{r}\right)$. Note that when $L=0, L^{\prime}=1$ we find the instantaneous model since $\overline{\mathbf{A}}_{\mathbf{x}}$ are no more matrices but scalars. Thus, it leads to the minimization of the following cost function:

$$
\mathcal{C}_{Z D}(\mathbf{B})=\sum_{i=1}^{N_{m}}\left\|\operatorname{Diag}\left\{\mathbf{B M}_{i} \mathbf{B}^{H}\right\}\right\|_{F}^{2},
$$

where $\mathbf{M}_{i}=\left(\overline{\mathbf{A}}_{\mathbf{x}}\right)_{i}$ is the $i-t h$ of the $N_{m}$ matrices belonging to $\mathcal{M}$. We suggest to use conjugate gradient and Levenberg-Marquardt algorithms [16], [17] to minimize the cost function given by Equation .(7) in order to estimate the matrix $\mathbf{B} \in \mathbb{C}^{n \times m}$. It means that $\mathbf{B}$ is re-estimated at each iteration $m$ (denoted $\mathbf{B}^{(m)}$ or $\mathbf{b}^{(m)}$ when the vector $\mathbf{b}^{(m)}=\operatorname{vec}\left(\mathbf{B}^{(m)}\right)$ is considered). The matrix $\mathbf{B}$ (or the vector $\mathbf{b}$ ) is updated according to the following adaptation rule for all $m=1,2, \ldots$ 


\section{Conjugate gradient approach}

$$
\left\{\begin{array}{l}
\mathbf{b}^{(m+1)}=\mathbf{b}^{(m)}-\mu^{(m)} \mathbf{d}_{\mathbf{B}}^{(m)} \\
\mathbf{d}_{\mathbf{B}}^{(m+1)}=-\mathbf{g}^{(m+1)}+\beta^{(m)} \mathbf{d}_{\mathbf{B}}^{(m)}
\end{array}\right.
$$

where $\mu$ is a positive small factor called the step-size, $\mathbf{d}_{\mathbf{B}}$ is the direction of search, $\beta$ is an exact line search and $\mathbf{g}=\operatorname{vec}\left(\nabla_{a} \mathcal{C}_{Z D}(\mathbf{B})\right)$ is the vectorization of the complex gradient matrix $\mathbf{G}=\nabla_{a} \mathcal{C}_{Z D}(\mathbf{B})=2 \sum_{i=1}^{N_{m}}\left[\operatorname{Diag}\left\{\mathbf{B M}_{i}\right.\right.$ $\left.\left.\mathbf{B}^{H}\right\} \mathbf{B M}_{i}^{H}+\left(\operatorname{Diag}\left\{\mathbf{B M}_{i} \mathbf{B}^{H}\right\}\right)^{H} \mathbf{B M}_{i}\right]$ (see the proof provided in [16] how the optimal step-size $\mu_{\mathrm{opt}}$, $\nabla_{a} \mathcal{C}_{Z D}(\mathbf{B})$ and $\beta$ are calculated at each iteration).

\section{Levenberg-Marquardt approach}

$$
\mathbf{b}^{(m)}=\mathbf{b}^{(m-1)}-\left[\mathbf{H}_{e}^{(m-1)}+\lambda \mathbf{I}_{m^{2}}\right]^{-1} \mathbf{g}^{(m-1)},
$$

where [.] $]^{-1}$ denotes the inverse of a matrix, $\lambda$ is positive a small damping factor, $\mathbf{I}_{m^{2}}$ is the $m^{2} \times m^{2}$ identity matrix, $\mathbf{H}_{e}=\left(\begin{array}{cc}\mathbf{H}_{e \mathbf{B}, \mathbf{B}^{*}}=\frac{\mathbf{A}_{00}+\mathbf{A}_{11}^{T}}{2} & \mathbf{H}_{e \mathbf{B}^{*}, \mathbf{B}^{*}}=\frac{\mathbf{A}_{01}+\mathbf{A}_{01}^{T}}{2} \\ \mathbf{H}_{e \mathbf{B}, \mathbf{B}}=\frac{\mathbf{A}_{10}+\mathbf{A}_{10}^{T}}{2} & \mathbf{H}_{e \mathbf{B}^{*}, \mathbf{B}}=\left[\mathbf{H}_{e \mathbf{B}, \mathbf{B}^{*}}\right]^{T}\end{array}\right)$ is the Hessian matrix of $\mathcal{C}_{Z D}(\mathbf{B})$ composed of four complex matrices with:

$$
\begin{aligned}
\mathbf{A}_{00} & =\left(\mathbf{M}_{i}^{T} \mathbf{B}^{T} \otimes \mathbf{I}_{N}\right) \mathbf{T}_{\text {Boff }}^{T}\left(\mathbf{B}^{*} \mathbf{M}_{i}^{*} \otimes \mathbf{I}_{N}\right)+\left(\mathbf{M}_{i}^{*} \mathbf{B}^{T} \otimes \mathbf{I}_{N}\right) \mathbf{T}_{\text {Boff }}^{T}\left(\mathbf{B}^{*} \mathbf{M}_{i}^{T} \otimes \mathbf{I}_{N}\right)+\mathbf{M}_{i}^{*} \otimes \operatorname{OffBdiag}_{(\mathbf{n})}\left\{\mathbf{B M}_{i} \mathbf{B}^{H}\right\} \\
& +\mathbf{M}_{i}^{T} \otimes \operatorname{OffBdiag}_{(\mathbf{n})}\left\{\mathbf{B} \mathbf{M}_{i}^{H} \mathbf{B}^{H}\right\}=\mathbf{A}_{11}^{*}, \\
\mathbf{A}_{10} & =\mathbf{K}_{N, M}^{T}\left(\mathbf{I}_{N} \otimes \mathbf{M}_{i} \mathbf{B}^{H}\right) \mathbf{T}_{\text {Boff }}^{T}\left(\mathbf{B}^{*} \mathbf{M}_{i}^{*} \otimes \mathbf{I}_{N}\right)+\mathbf{K}_{N, M}^{T}\left(\mathbf{I}_{N} \otimes \mathbf{M}_{i}^{H} \mathbf{B}^{H}\right) \mathbf{T}_{\text {Boff }}^{T}\left(\mathbf{B}^{*} \mathbf{M}_{i}^{T} \otimes \mathbf{I}_{N}\right)=\mathbf{A}_{01}^{*},
\end{aligned}
$$

where the operator $\otimes$ denotes the Kronecker product, $\mathbf{K}_{N, M}$ is a square commutation matrix of size $N M \times N M$ and $\mathbf{T}_{\text {Boff }}=\mathbf{I}_{N^{2}}-\mathbf{T}_{\text {Diag }}$, is the $N^{2} \times N^{2}$ "transformation" matrix, with $\mathbf{T}_{\text {Diag }}=\operatorname{diag}\left\{\operatorname{vec}\left(\operatorname{BDiag}\left\{\mathbf{1}_{N}\right\}\right)\right\}$, $\mathbf{1}_{N}$ is the $N \times N$ matrix whose components are all ones, $\operatorname{diag}\{\mathbf{a}\}$ is a square diagonal matrix whose diagonal elements are the elements of the vector $\mathbf{a}, \mathbf{I}_{N^{2}}=\operatorname{Diag}\left\{\mathbf{1}_{N^{2}}\right\}$ is the $N^{2} \times N^{2}$ identity matrix, $\operatorname{and} \operatorname{Diag}\{\mathbf{A}\}$ is the square diagonal matrix with the same diagonal elements as $\mathbf{A}$.

\subsection{Summary of the proposed methods}

The proposed methods $J Z D_{C G_{D D}}$ and $J_{Z D_{L M}}$ combine the NU - JZD algorithms which are JZD and $J Z D_{\mathrm{LM}}$ together with the detector $C_{\text {Ins }}$. Its principles are summarized below:

Data: Consider the $N_{m}$ matrices of set $\mathcal{M}:\left\{\left(\overline{\mathbf{A}}_{\mathbf{x}}\right)_{1},\left(\overline{\mathbf{A}}_{\mathbf{x}}\right)_{2}, \ldots,\left(\overline{\mathbf{A}}_{\mathbf{x}}\right)_{N_{m}}\right\}$, stopping criterion $\epsilon$, step-size $\mu$ (for conjugate gradient), max. number of iterations $M_{\max }$

Result: Estimation of joint zero diagonalizer $\mathbf{B}$

initialize: $\mathbf{B}^{(0)} ; \lambda^{(0)} ; m=0 ; \mathbf{D}^{(0)}$ (for conjugate gradient);
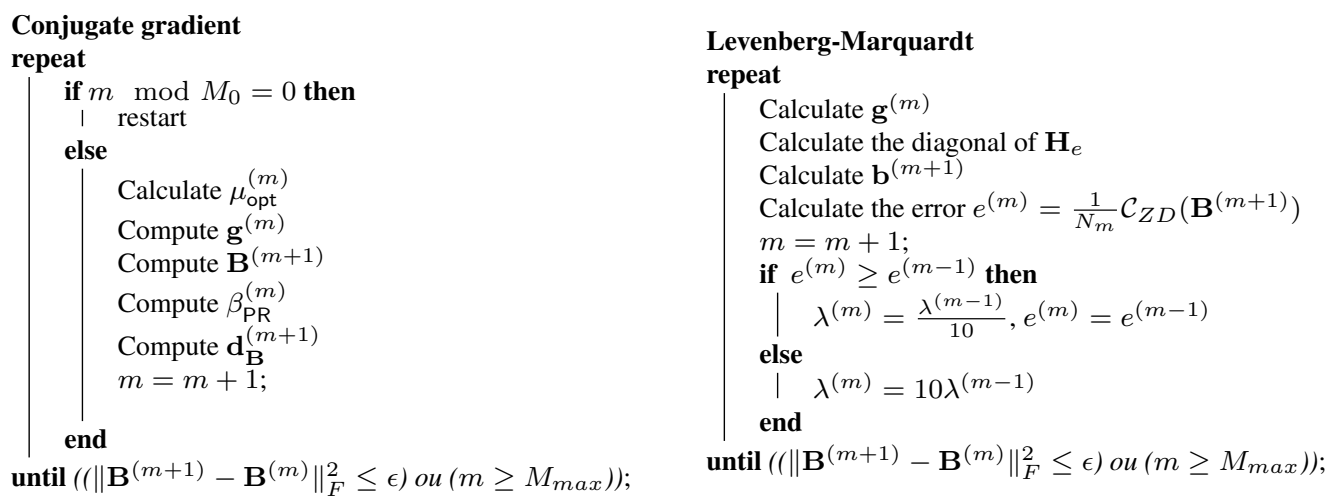

\section{COMPUTER SIMULATIONS}

Computer simulations are performed to illustrate the good behavior of the suggested methods and to compare them with the same kind of existing approach denoted by JZD Chabriel $_{D D}$ proposed in [24] with the 
Delay-Doppler point $C_{\text {Ins }}$ detector. We consider $m=3$ mixtures of $n=2$ frames of 256 bits correspond to two vessels with different characteristics. The frames are generated according to the S-AIS recommendation as mentioned in the Figure 2 (see also [11], [10]). These frames are encoded with NRZI and modulated in GMSK with a bandwidth-bit-time product parameter $\mathrm{BT}=0.4$. The transmission bit rate is $=9600 \mathrm{bps}$ and the order gaussian filter is $\mathrm{OF}=21$. The frequency carrier of the first source (resp. the second source) is $161.975 \mathrm{MHz}$ (resp. $162.025 \mathrm{MHz}$ ), taking into account a delay of $10 \mathrm{~ms}$ and a Doppler shift of $4 \mathrm{kHz}$ (resp. a delay of $0 \mathrm{~ms}$ and the Doppler shift of $0 \mathrm{~Hz}$ ). These sources correspond to 1400 time samples which are mixed according to a mixture matrix $\mathbf{H}$ whose components stands for:

$$
\mathbf{H}=\left(\begin{array}{cc}
-1.1974 & 1.3646 \\
0.8623 & 1.6107 \\
0.1568 & -0.9674
\end{array}\right)
$$

The real part and the imaginary part of their SGMAF is given on the left and on the right of the Figure 4 respectively. Then, the SGMAF of the observations $\mathrm{x}$ is then calculated by (5) and finally the 100 resulting SGMAF are averaged. We have chosen $\epsilon_{1}=0.07$ and $\epsilon_{2}=0.08$ for the detector $C_{\operatorname{lns}}$ in order to construct the set $\mathcal{M}$ to be joint zero-diagonalized. The signal-to-noise ratio SNR is defined by SNR $=10 \log \left(\frac{1}{\sigma_{N}^{2}}\right)$ of mean 0 and variance $\sigma_{n}^{2}$. The selected Delay-Doppler points using the proposed detector are represented in the Figure 5 for $\mathrm{SNR}=10 \mathrm{~dB}$ and $100 \mathrm{~dB}$.
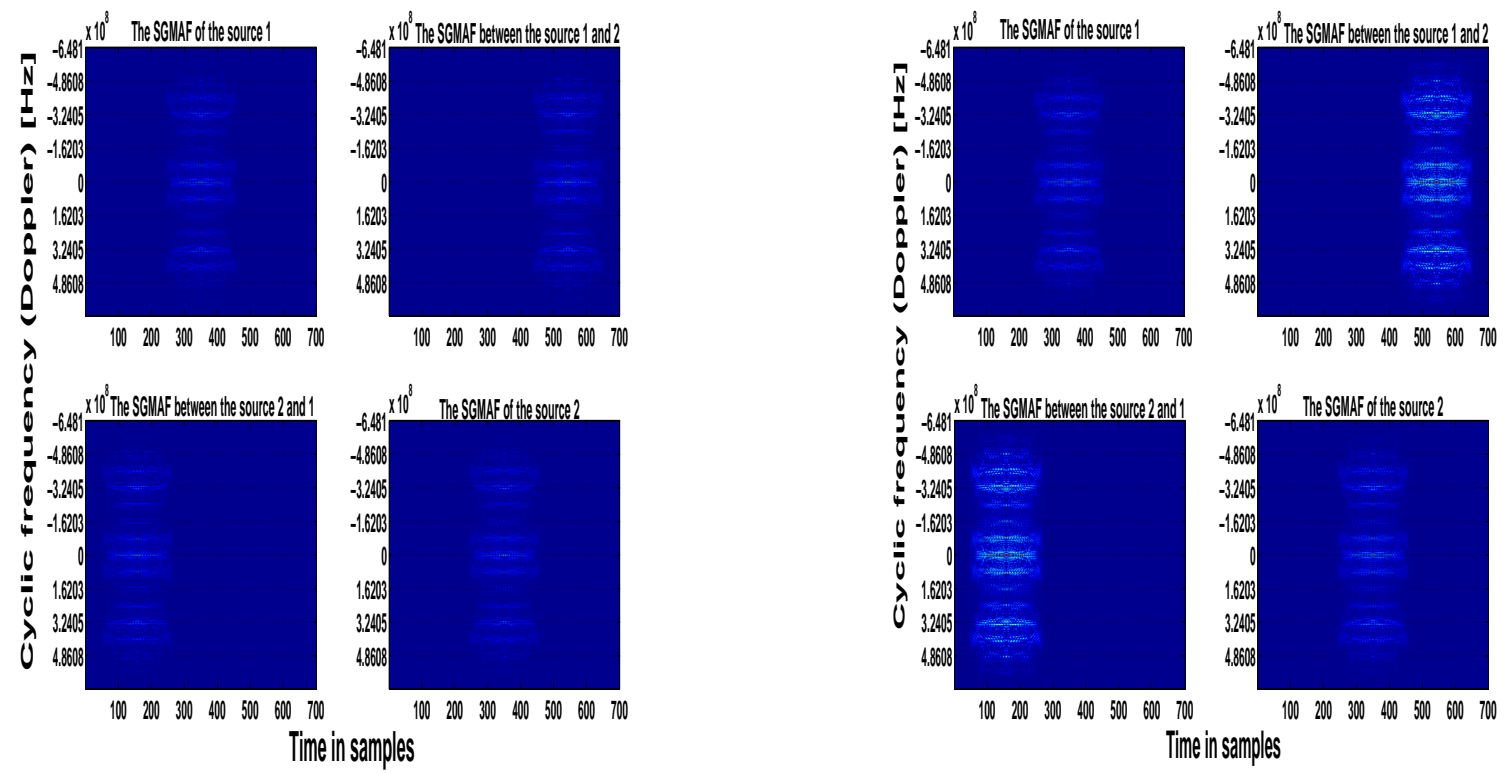

Figure 4. Left : The SGMAF real part of the S-AIS sources. Right : The SGMAF imaginary part of the S-AIS sources.

To measure the quality of the estimation, the ensuing error index is used [25] :

$$
I(\mathbf{T})=\frac{1}{n(n-1)}\left[\sum_{i=1}^{n}\left(\sum_{j=1}^{n} \frac{\left\|T_{i, j}\right\|_{F}^{2}}{\max _{\ell}\left\|T_{i, \ell}\right\|_{F}^{2}}-1\right)+\sum_{j=1}^{n}\left(\sum_{i=1}^{n} \frac{\left\|T_{i, j}\right\|_{F}^{2}}{\max _{\ell}\left\|T_{\ell, j}\right\|_{F}^{2}}-1\right)\right],
$$

where $(T)_{i, j}$ for all $i, j \in 1, \ldots, n$ is the $(i, j)$-th element of $\mathbf{T}=\hat{\mathbf{B}} \mathbf{H}$. The separation is perfect when the error index $I(\cdot)$ is close to 0 in a linear scale $(-\infty$ in a logarithmic scale). All the displayed results have been averaged over 30 Monte-Carlo trials. We plot, in the Figure 6, the evolution of the error index versus the SNR in order to emphasize the influence of this in the quality of the estimation. All algorithms are initialized using the same initialization suggested in [24]. 
First, we can deduce from the Figure 4 that the diversity in the Delay-Doppler regions is obtained on the zero-diagonal part which supports the use of zero diagonalization algorithms. Then, our analysis are examined on the Figure 6 according to noiseless and noisy contexts . For the noiseless context (when SNR=100 $\mathrm{dB}$ ), the $J_{Z D_{C_{D D}}}$ and $J Z D_{L M_{D D}}$ reach approximately $-64 \mathrm{~dB}$ and -60 comparing with $J_{Z D_{C h a b r i e l}}$ method which reaches $\simeq-20 \mathrm{~dB}$. From this comparison, we have checked the validity of the good behavior of $J Z D_{C G_{D D}}$ and JZD $\mathrm{LM}_{\mathrm{DD}}$ compared to the JZD $\mathrm{Chabrie}_{\mathrm{DD}}$ approach. Moreover, we observe that the JZD $\mathrm{LM}_{\mathrm{DD}}$ based on the computation of exact Hessian matrices is more efficient than the $\mathrm{JZD}_{\mathrm{CG}_{\mathrm{DD}}}$ approach. Even in a difficult (noisy) context (for example $S N R=15 \mathrm{~dB}$ ), we note that the best results are generally obtained using the JZD $D_{L M_{D D}}$ $(-36 \mathrm{~dB})$ then $J Z D_{C G_{D D}}(-33 \mathrm{~dB})$ especially the $J_{Z D_{L M}}$ algorithm based on the computation of exact Hessian matrices. It may be concluded that the approaches exploiting the Delay-Doppler diversity of S-AIS signals seem rather promising. Due to its robustness to the noise, it seems to be able to solve the problem of BSS (i.e the collision problem) in a marine surveillance context.
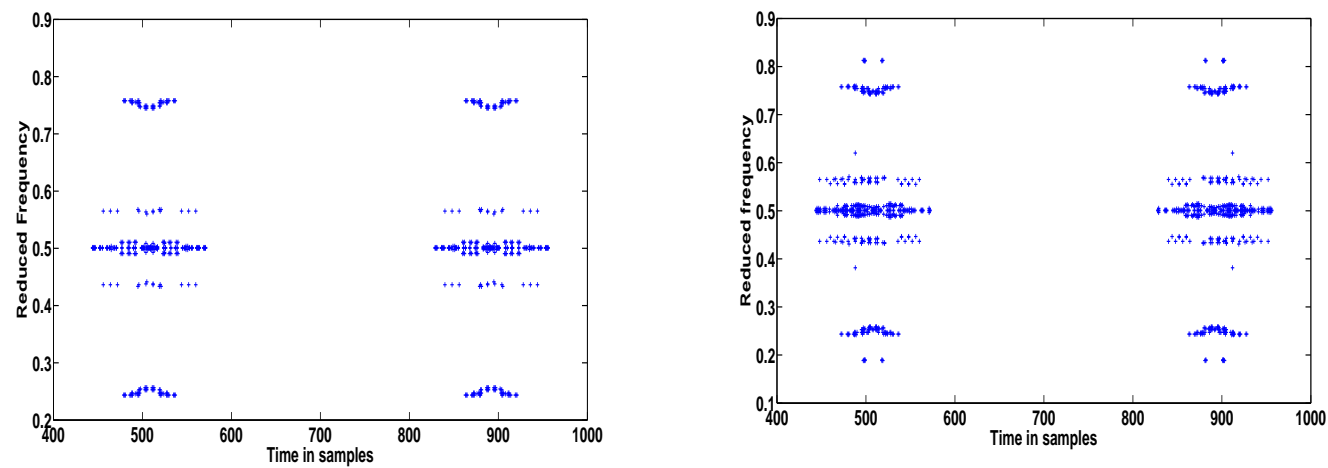

Figure 5. Delay-Doppler points selected with the detector $C_{\text {Ins }}$. left : $S N R=100 \mathrm{~dB}$. right : $S N R=10 \mathrm{~dB}$.

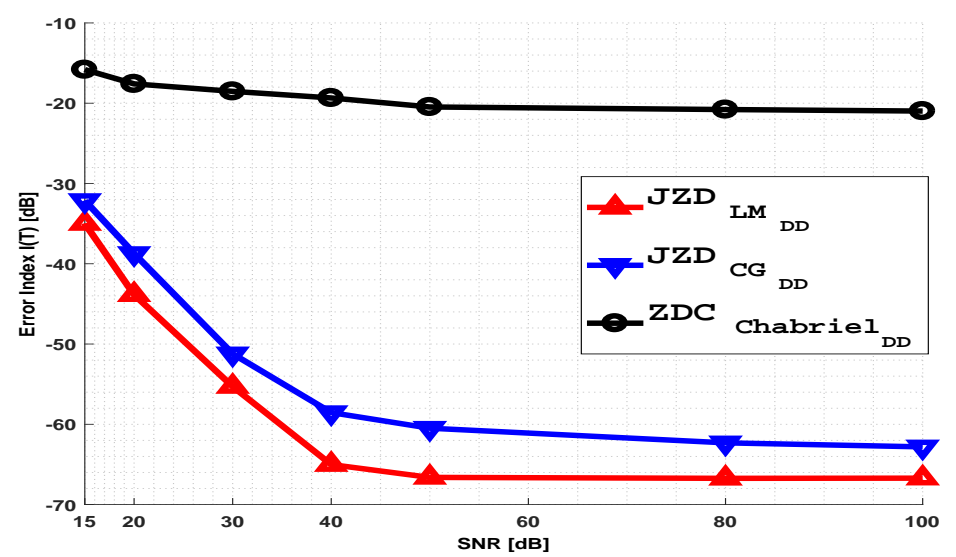

Figure 6. Comparison of the different methods: evolution of the error index $I(\mathbf{T})$ in $\mathrm{dB}$ versus SNR.

\section{CONCLUSION}

In this paper, we have shown that the blind source separation based on SGMAF can be performed. We have considered complex-valued S-AIS data for marine surveillance which can be received at the same timeslot in where the collision of these data is caused. In addition, it is presented that the collision problem can be reshaped into BSS problem. Moreover, it is shown that proposed BSS methods are established thanks to an automatic single cross-term selection procedure combined with two NU - JZD algorithms denoted Conjugate Gradient and Levenberg-Marquardt which are based on the minimization of a least-mean-square criterion. Finally, we deduced that the $J Z D_{L M_{D D}}$ and $J Z D_{C G_{D D}}$ offer the best performances even in noisy contexts. As perspective, a question needing analysis is to study more realistic and complex cases in which the number of S-AIS messages received at the antenna embedded in the satellite would be much higher and mixing models could also be considered. 


\section{REFERENCES}

[1] B. Tetreault, "Use of the automatic identification system (ais) for maritime domain awareness (mda)," in OCEANS, 2005. Proceedings of MTS/IEEE, vol. 2, Sep 2005, pp. 1590-1594.

[2] International Association of Maritime Aids to Navigation and Lighthouse Authorities (IALA), IALA guidelines on the universal automatic identification system (AIS). IALA, 2002.

[3] G. Høye, B. Narheim, T. Eriksen, and B. J. Meland, Euclid JP 9.16, Space-Based AIS Reception for Ship Identification. FFI, 2004.

[4] T. Wahl and G. Høye, "New possible roles of small satellites in maritime surveillance," Acta Astronautica, vol. 56, no. 1-2, pp. 273-277, 2005.

[5] T. Eriksen, G. Høye, B. Narheim, and B. Meland, "Maritime traffic monitoring using a space-based ais receiver," Acta Astronautica, vol. 58, no. 10, pp. 537-549, May 2006.

[6] P. Burzigotti, A. Ginesi, and G. Colavolpe, "Advanced receiver design for satellite-based ais signal detection," in Advanced satellite multimedia systems conference and the 11th signal processing for space communications workshop, Sep 2010, pp. 1-8.

[7] International Telecommunications Union Recommendation ITU-R M.2169, Improved Satellite Detection of AIS, Dec 2009.

[8] J. Tunaley, “An analysis of ais signal collisions," Tech. Rep., Aug 2005.

[9] M. Yang, Y. Zou, and L. Fang, "Collision and detection performance with three overlap signal collisions in space-based ais reception," in Trust, Security and Privacy in Computing and Communications (TrustCom), 2012 IEEE 11th International Conference on, Jun 2012, pp. 1641-1648.

[10] R. Prevost, M. Coulon, D. Bonacci, J. LeMaitre, J.-P. Millerioux, and J.-Y. Tourneret, "Interference mitigation and error correction method for ais signals received by satellite," in Signal Processing Conference (EUSIPCO), 2012 Proceedings of the 20th European, 2012, pp. 46-50.

[11] M. Zhou, A.-J. van der Veen, and R. Van Leuken, "Multi-user leo-satellite receiver for robust space detection of ais messages," in Acoustics, Speech and Signal Processing (ICASSP), 2012 IEEE International Conference on, Mar 2012, pp. 2529-2532.

[12] A.-J. van der Veen and A. Paulraj, "An analytical constant modulus algorithm," Signal Processing, IEEE Transactions on, vol. 44, no. 5, pp. 1136-1155, May 1996.

[13] L. Chang, "Study of ais communication protocol in vts," in Signal Processing Systems (ICSPS), 2010 2nd International Conference on, vol. 1, Jul 2010, pp. 168-171.

[14] A. Linz and A. Hendrickson, "Efficient implementation of an i-q gmsk modulator," IEEE Trans. Circuits Syst. II, Analog Digit. Signal Process., vol. 43, no. 1, Jan 1996.

[15] R. Prevost, M. Coulon, D. Bonacci, J. LeMaitre, J.-P. Millerioux, and J.-Y. Tourneret, "Joint phaserecovery and demodulation-decoding of ais signals received by satellite," in Acoustics, Speech and Signal Processing (ICASSP), 2013 IEEE International Conference on, May 2013, pp. 4913-4917.

[16] O. Cherrak, H. Ghennioui, N. Thirion-Moreau, E.-H. Abarkan, and E. Moreau, "Non-unitary joint zeroblock diagonalization of matrices using a conjugate gradient algorithm," in Proc. EUSIPCO, Sep 2015.

[17] O. Cherrak, H. Ghennioui, N. Thirion-Moreau, and E.-H. Abarkan, "Nouvel algorithme de zéro-bloc diagonalisation conjointe par approche de levenberg-marquardt," in 25ème Colloque GRETSI, Lyon, France, Sep 2015.

[18] A. Belouchrani and M. Amin, "Blind source separation based on time-frequency signal representations," Signal Processing, IEEE Transactions on, vol. 46, no. 11, pp. 2888-2897, Nov 1998.

[19] M. G. Amin, "Blind time-frequency analysis for source discrimination in multisensor array processing," Villanova University, Department of Electrical and Computer Engineering, Technical Report, Oct 1998.

[20] F. Hlawatsch and F. Auger, Eds., Temps-fréquence et traitement statistique. Hermès Sciences Publications, 2005.

[21] W. Kozek, "On the transfer function calculus for underspread ltv channels," IEEE Trans. Signal Proc., vol. 45, no. 1, Jan 1997.

[22] _ - "Matched weyl-heisenberg expansions of nonstationary environments," Ph.D. dissertation, Vienna University of Technology, 1997.

[23] E. Fadaili, N. Moreau, and E. Moreau, "Nonorthogonal joint diagonalization/zero diagonalization for source separation based on time-frequency distributions," IEEE Trans. Signal Proc., vol. 55, no. 5, pp. 1673-1687, May 2007.

[24] G. Chabriel, J. Barrere, N. Thirion-Moreau, and E. Moreau, "Algebraic joint zero-diagonalization and 
blind sources separation,” IEEE Trans. Signal Proc., vol. 56, no. 3, pp. 980-989, Mar 2008.

[25] E. Moreau, "A generalization of joint-diagonalization criteria for source separation," IEEE Trans. Signal Proc., vol. 49, no. 3, pp. 530-541, Mar 2001.

\section{BIOGRAPHIES OF AUTHORS}
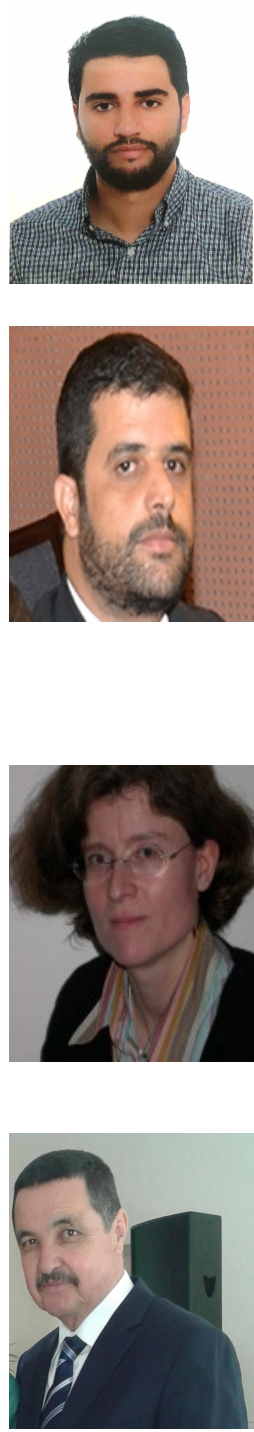

Omar Cherrak was born in Fez Morocco. He received the Bachelor degree in 2009 in the field of Electronics Telecommunications and Computer Sciences and the Master degree of Microelectronic, Telecommunications and Computer Industry Systems in 2011, both from the Université Sidi Mohamed Ben Abdellah (USMBA), Faculté des Sciences et Techniques (FST), Fez Morocco. He obtained his Ph.D. degree on March 2016 in the area of "Signal, Telecommunications, Image and Radar" from Université de Toulon and this thesis was carried out in cotutelle with USMBA. His main research interests are blind source separation, telecommunications, joint matrix decompositions, maritime surveillance system, time-frequency representation, smart grid and DoA estimation.

Hicham Ghennioui obtained the Maîtrise degree in Telecommunications from the Faculty of Sciences and Technologies (FST), Fez, Morocco, in 2002. He got the D.E.S.A. degree in Computer and Telecommunications from the Faculty of Sciences, Rabat, Morocco, in 2004 and the $\mathrm{Ph} . \mathrm{D}$ degree in engineering sciences in 2008, from Mohamed V - Agdal University, Morocco, and the University of Toulon, France, respectively. From May 2008 to December 2009, he was a Research \& Development Engineer at Amesys Bull, and form January 2010 to May 2011, he was a Signal/Image Researcher at Moroccan foundation for Advanced Science, Innovation and Research (MASCIR), Rabat, Morocco. Since 2011, he is an Assistant Professor at the Electrical Engineering Department of the Faculty of Sciences and Techniques, Sidi Mohamed Ben Abdellah University, Fez, Morocco. His main research interests are signal/image processing including blind sources separation, deconvolution, deblurring, time-frequency representations and cognitive radio.

Nadège Thirion-Moreau was born in Montbéliard France. She received the DEA degree in 1992 and the Ph.D. degree in 1995, both in the field of signal processing and from the Ecole Nationale Supérieure de Physique (ENSPG) Institut National Polytechnique de Grenoble (INPG), France. From 1996 to 1998, she was an Assistant Professor at the Ecole Supérieure des Procédés Electroniques et Optiques (ESPEO), Orléans, France. Since 1998, she has been with the Institut des Sciences de l'Ingénieur de Toulon et du Var (ISITV), La Valette, France, as an Assistant Professor, in the Department of Telecommunications. Her main research interests are in deterministic and statistical signal processing including array processing, blind sources separation/equalization, highorder statistics, nonstationary signals, time-frequency representations, and decision/ classification.

El Hossain Abarkan was born in 1953, Nador, Morocco. He graduated the bachelor degree in physics from Mohammed V University, Rabat, Morocco, in 1978. He obtained the DEA, the Doctorate and the Ph.D degrees from University of Languedoc, Montpellier, France, in 1979, 1981 and 1987 respectively. He got the Ph.D degree from the University of Sidi Mohamed Ben Abdellah, Fez, Morocco, in 1992. Currently, he is a Professor in Sidi Mohamed Ben Abdellah University. From 1996 to 2014, he was the founder and the director of the Laboratory of Signals, Systems and Components, Sidi Mohamed Ben Abdellah University. His main research interests are in electronics, Modeling, characterization and CAD in integrated circuits. 